\title{
Simulation studies on the tomographic reconstruction of the equatorial and low-latitude ionosphere in the context of the Indian tomography experiment: CRABEX
}

\author{
S. V. Thampi, T. K. Pant, S. Ravindran, C. V. Devasia, and R. Sridharan \\ Space Physics Laboratory, Vikram Sarabhai Space Centre Trivandrum, 695 022, India \\ Received: 17 March 2004 - Revised: 9 June 2004 - Accepted: 18 June 2004 - Published: 3 November 2004
}

\begin{abstract}
Equatorial ionosphere poses a challenge to any algorithm that is used for tomographic reconstruction because of the phenomena like the Equatorial Ionization Anomaly (EIA) and Equatorial Spread F (ESF). Any tomographic reconstruction of ionospheric density distributions in the equatorial region is not acceptable if it does not image these phenomena, which exhibit large spatial and temporal variability, to a reasonable accuracy. The accuracy of the reconstructed image generally depends on many factors, such as the satellite-receiver configuration, the ray path modelling, grid intersections and finally, the reconstruction algorithm. The present simulation study is performed to examine these in the context of the operational Coherent Radio Beacon Experiment (CRABEX) network just commenced in India. The feasibility of using this network for the studies of the equatorial and low-latitude ionosphere over Indian longitudes has been investigated through simulations. The electron density distributions that are characteristic of EIA and ESF are fed into various simulations and the reconstructed tomograms are investigated in terms of their reproducing capabilities. It is seen that, with the present receiver chain existing from $8.5^{\circ} \mathrm{N}$ to $34^{\circ} \mathrm{N}$, it would be possible to obtain accurate images of EIA and the plasma bubbles. The Singular Value Decomposition (SVD) algorithm has been used for the inversion procedure in this study. As is known, by the very nature of ionospheric tomography experiments, the received data contain various kinds of errors, like the measurement and discretization errors. The sensitivity of the inversion algorithm, SVD in the present case, to these errors has also been investigated and quantified.
\end{abstract}

Key words. Ionospheric (equatorial ionosphere; ionospheric irregularities; instruments and techniques)

Correspondence to: $\mathrm{S}$. V. Thampi

(sudha_ravindran@vssc.org)

\section{Introduction}

The basic idea of tomographic methods, which uses the line integral measurements through a field to reconstruct the field itself, was originated by Radon in 1917 and the first practical application seems to have been published by Bracewell (1956). Since then, this technique has been applied successfully in many fields. For instance, it had a revolutionary impact in the field of medical imaging. Austen et al. (1988) suggested that using the total electron content (TEC), the tomography technique can also be used for the two-dimensional imaging of electron density distribution in the ionosphere.

The first experimental test of the application of tomographic methods for ionospheric studies was attempted using coordinated observations in Scandinavia in the year 1986 (Andreeva et al., 1990; Pryse and Kersley, 1992). Simultaneous TEC measurements were made from two stations by receiving phase coherent signals of $150 \mathrm{MHz}$ and $400 \mathrm{MHz}$ transmitted from a low Earth-orbiting Navy Navigational Satellite. Despite all the limitations of the experimental arrangement, the tomographic image could reveal the presence of a gradual, large-scale, northward electron density gradient, which was confirmed by independent measurements using the EISCAT radar (Raymund et al., 1993). Subsequently, several experiments were carried out in both the United Kingdom and Scandinavia, which demonstrated the potential of tomography to image large-scale ionospheric, features such as troughs in electron density found at auroral and subauroral latitudes (Kersley et al., 1997). Apart from these, there were tomographic experiments from other parts of the world also. The most important of them include the Russian Tomography Experiments, Russian-American Tomography Experiment (RATE) and the Mid-America Computerized Ionospheric tomography Experiment (MACE) (Andreeva et al., 1990, 1992; Kunitsyn and Treshchenko, 1994; Foster et al., 1994). All these experiments could bring out the shape, width, slope and depth of the main ionospheric trough very clearly. These experiments could also image the 
traveling ionospheric disturbances (TIDs) and quasi-wave structures which usually appear in the perturbed ionosphere.

It has been unambiguously established now that the tomographic techniques are very effective and useful in investigating the large-scale structures over low and equatorial latitudes, for example, equatorial ionization anomaly (EIA) (Andreeva et al., 2000). The first network of six receivers from $14.6^{\circ} \mathrm{N}$ to $31.3^{\circ} \mathrm{N}$ (geographic latitudes) along the $121^{\circ} \mathrm{E}$ meridian, namely the Low latitude Ionospheric Tomography Network (LITN), has provided important information about the motion of the anomaly crest of the EIA (Yeh et al., 1994, 2001), the structure and symmetry of its core (Andreeva et al., 2000) and the low-latitude ionospheric response to magnetic storms (Ji-Sheng et al., 2000). However, questions regarding the development of the anomaly during the course of the day, and its relationship with other ionospheric processes, like the Equatorial electrojet and equatorial spread $\mathrm{F}$, calls for further systematic investigations.

The Indian Coherent Radio Beacon Experiment (CRABEX) has been just initiated mainly to address the aforementioned questions regarding the large-scale processes over equatorial and low latitudes. This experiment consists of six radio receivers stationed at Trivandrum $\left(8.5^{\circ} \mathrm{N}, 77^{\circ} \mathrm{E}\right)$, Bangalore $\left(13^{\circ} \mathrm{N}, 77.6^{\circ} \mathrm{E}\right)$, Hyderabad $\left(17.3^{\circ} \mathrm{N}, 78.3^{\circ} \mathrm{E}\right)$, Bhopal $\left(23.2^{\circ} \mathrm{N}, 77.2^{\circ} \mathrm{E}\right)$, Delhi $\left(28.8^{\circ} \mathrm{N}, 77.2^{\circ} \mathrm{E}\right)$, and Hanle $\left(34^{\circ} \mathrm{N}, 78^{\circ} \mathrm{E}\right)$ that are capable of receiving the 150 and $400 \mathrm{MHz}$ beacon transmissions from the Low Earth Orbiting Satellites (LEOS). This chain is unique as it covers the crest and trough regions of the EIA latitudinally, and goes well beyond the anomaly region. Thus, the data obtained using this chain will help in understanding the temporal and spatial evolution of equatorial and low-latitude ionospheric phenomena like EIA and ESF, and their interrelationships. For example, the degree of variability of the EIA, which is otherwise quite difficult to be monitored completely, can be understood by the tomographic methods. The maximum $\boldsymbol{E} \times \boldsymbol{B}$ drift, the location of the EIA crest and its intensity which are known to be intricately connected to the generation of equatorial bubbles, as well as bottomside spread F (Whalen, 2001), can be investigated in detail using the tomograms.

The measured parameter in most of the ionospheric tomography experiments worldwide is the relative phase of $150 \mathrm{MHz}$ signal with respect to that of $400 \mathrm{MHz}$, both transmitted coherently from a satellite borne-beacon and received on ground. The differential phase is related to the slant electron content along any ray as

$\Phi=C_{D} \times S T E C$,

where $\Phi$ is measured in radians, STEC is in $\mathrm{m}^{-2}$ and $\mathrm{C}_{D}=$ $1.6132 \times 10^{-15}$ for NNSS satellites (Leitinger, 1994).

The TEC is estimated along a number of ray paths that define the passage of a low Earth-orbiting satellite (LEOS) as seen by the ground-based receiver at a given location. Thus, estimated TECs for a chain of receivers are then inverted to obtain the electron density distribution as a function of latitude and altitude over a given longitude. Inversion, by nature being an ill-defined mathematical problem, does not always have a unique set of solutions. As a result, various algorithms are used for this inversion. In ionospheric tomography, when used to reconstruct the electron density profiles, almost all of these algorithms suffer from the basic inability to correctly estimate the vertical profiles of these distributions. In other words, no particular algorithm is able to give a unique solution to the problem of ionospheric tomography. This problem to a large extent is also due to the geometry of computerized ionospheric tomography (CIT) systems, where one cannot have TEC information from large projection angles for a given ground-based receiver. The completeness of the data is limited, as the receivers do not lock onto a satellite beacon until it is at least a few degrees above the horizon. The present tomography network gives $10-12 \mathrm{~min}$. of data for a high elevation pass ( $>60 \mathrm{deg}$.), from each receiver but the simultaneous data, for all five receivers will be only for 5-6 min, and the data from each receiver is incomplete due to the lack of data from large projection angles.

As a fundamental principle in inversion problems, there is a direct relationship between the information contained in the measured data and the accuracy of the reconstructed image (Na et al., 1995). This means that the proper choice of the locations of the receiving stations, which could optimize the information content in a given latitudinal plane is one of the key factors for obtaining accurate images from any ionospheric tomography network. Further, the location of the receivers also depends on the availability of suitable sites. So, an extensive feasibility study using known theoretical/model generated electron density distributions is extremely useful in optimizing the receiver chain, to give reliable ionospheric images, especially when the region of interest is replete with large-scale ionospheric processes, like the Equatorial Ionization Anomaly and Equatorial Spread F. The present paper describes the results of one such feasibility study conducted considering the receiver chain recently established for the Indian tomography experiment. It presents only the simulations, not actual data, as the present receiver chain is still undergoing modifications. Nonetheless, tomographic images obtained using CRABEX data will be presented in the future.

\section{Inversion problem and algorithms used}

As mentioned earlier, the inversion algorithm plays a very important role in the retrieval of the electron density distribution, the latitudinal and altitudinal extents of ionospheric structures if present, and their magnitudes and peak positions. Any good algorithm would be able to image these characteristics within the limitation of the resolution used in the forward model. Numerous inversion algorithms/methods and their modifications have been tried out for this purpose and the details are available in the literature. The most conventional ones are the iterative methods, like Algebraic Reconstruction Technique (ART), Multiplicative Algebraic Reconstruction Technique (MART), and Simultaneous Iterative Reconstruction Technique (SIRT) (Andreeva et al., 1992; 
Censor, 1983; Raymund et al., 1990), as well as the maximum entropy method (Pakula et al., 1995; Fougere, 1995). In these algorithms the iteration begins from a chosen initial profile and proceeds step by step until a given stop criterion is met. The improper choice of the initial guess would produce somewhat erroneous results. To avoid such errors, other techniques are used wherein the inversion is done in a single step with suitable matrix operations. The reconstruction using Singular Value Decomposition (SVD) falls under this category. The SVD algorithm and its modifications are already used for several experimental as well as theoretical studies of tomography ( $\mathrm{Na}$ and Sutton, 1994; Hajj et al., 1994; Kunitake et al., 1995; Zhou et al., 1999). SVD, being a non-iterative technique does not require any initial guess and is especially suitable in understandinging the effects of receiver configuration on the accuracy of the reconstructed images. The present feasibility study also uses the SVD technique for obtaining the tomograms. The uniqueness of this study lies not in the SVD technique itself, but in our use of this technique to understand the capability of the newly established Indian tomographic chain in terms of reproducing the features present in the equatorial ionosphere. Mathematical preliminaries of this technique, as employed in an ionospheric tomography problem, are given in the next section.

\section{Basic theory of SVD}

The ionospheric tomography problem can be expressed mathematically by a set of simple linear algebraic equations as follows: (Fremouw et al., 1992, 1994)

$Y=A x+E$,

where, $\mathrm{Y}$ is a vector of the observed TEC data, $\mathrm{x}$ is a vector of the unknown electron densities, and $\mathrm{A}$ is the geometry matrix, which describes the relationship between the received TEC data and the electron densities on each ray path. The "noise vector" E represents the measurement and discretization errors.

The problem of ionospheric tomography is basically ill posed, such that the nature of the problem is mathematically manifested by the non-existence of the inverse matrix $\left(\mathrm{A}^{-1}\right)$, as the problem can be either underdetermined or overdetermined. Any reconstruction algorithm should be able to tackle this ill-posed nature of the problem, to give the best approximate solution that is consistent with the experimental data. So here we look for a matrix $\mathrm{A}^{-g}$, which is called the generalized inverse, which can be computed with the help of SVD in that any real $(m \times n)$ matrix can be decomposed as

$A=U D V^{T}$

where $\mathrm{U}$ is a real $(\mathrm{m} \times \mathrm{k})$ matrix with orthonormal columns, $\mathrm{V}$ is a real $(\mathrm{n} \times \mathrm{k})$ matrix with orthonormal columns, and D is a real $(\mathrm{k} \times \mathrm{k})$ diagonal positive matrix, which contains the singular values $\left(\mathrm{sv}_{i}\right)$ of the matrix $\mathrm{A}$ and $\mathrm{k}=\min (\mathrm{m}, \mathrm{n})$.
When $\mathrm{A}$ is of full rank, $\mathrm{sv}_{i}>0$. If $\mathrm{A}$ is rank deficient, (as in the case of tomography problem), there are singular values that are equal to zero. Essentially, the vectors corresponding to non-zero singular values span the information space, while the other vectors corresponding to zero singular values span the null space. In this rank deficient case, SVD computes the diagonal matrix $\mathrm{D}^{-g}$ with elements $\mathrm{d}^{-g}$ as

$$
\begin{aligned}
& \mathrm{d}_{i}^{-g}=\mathrm{sv}_{i}^{-1} \text { if } \mathrm{sv}_{i}>0, \\
& \text { and } \mathrm{d}_{i}^{-g}=0 \text { if } \mathrm{sv}_{i}=0, \text { so that }
\end{aligned}
$$

$A^{-g}=V D^{-g} U^{T}$

Therefore, SVD provides a direct method to identify the information space (data space) and the undeterminable space or the null space (Zhou et al., 1999). In other words, SVD avoids the undeterminable parts, which would otherwise give rise to infinities in the solution, and thus, it provides a powerful tool for this linear inverse problem (Press et al., 1989).

In the solutions given by SVD, the smallest singular values have the largest effect, by weight of their large inverses, $\mathrm{sv}_{i}^{-1}$, in $\mathrm{D}^{-g}$. This applies to both the error free and error components of the data.

When the data contains errors, the magnifying effect of the smaller singular values can cause significant instability to the solution. This means that the smaller singular values cause larger perturbations in the solution. Truncation is used to filter out such perturbations. This is a simple regularization strategy applied for stabilizing the solution of the ill-posed inverse problem. In other words, the norm of the solution is minimized by neglecting some of the smallest singular values. Essentially, this means that we seek the solution of the following problem,

$\min _{x \in b}\|x\|_{2}, b=\left\{x \mid\left\|A_{l} x-Y\right\|_{2}=\min \right\}$,

where only $l$ singular values are used, and $Y$ represents the data vector with noises.

This truncated SVD (TSVD) is useful in actual experimental situations, where the data will always contain various kinds of errors (Kunitake et al., 1995).

The SVD-based formalism is particularly appropriate for understanding the interrelationship between the configuration of measurement links (receivers), the data noise and the basis functions, which correspond to the particular geometry involved in the problem. Thus, it is an ideal method for analyzing the effects of receiver locations, in the reconstruction in relation to the various ionospheric structures that could be present in the medium.

\section{Present study}

Figure 1 shows a typical LEO satellite and ground-based receiver configuration used for ionospheric tomography. For clarity sake only three receivers are shown on the ground, 


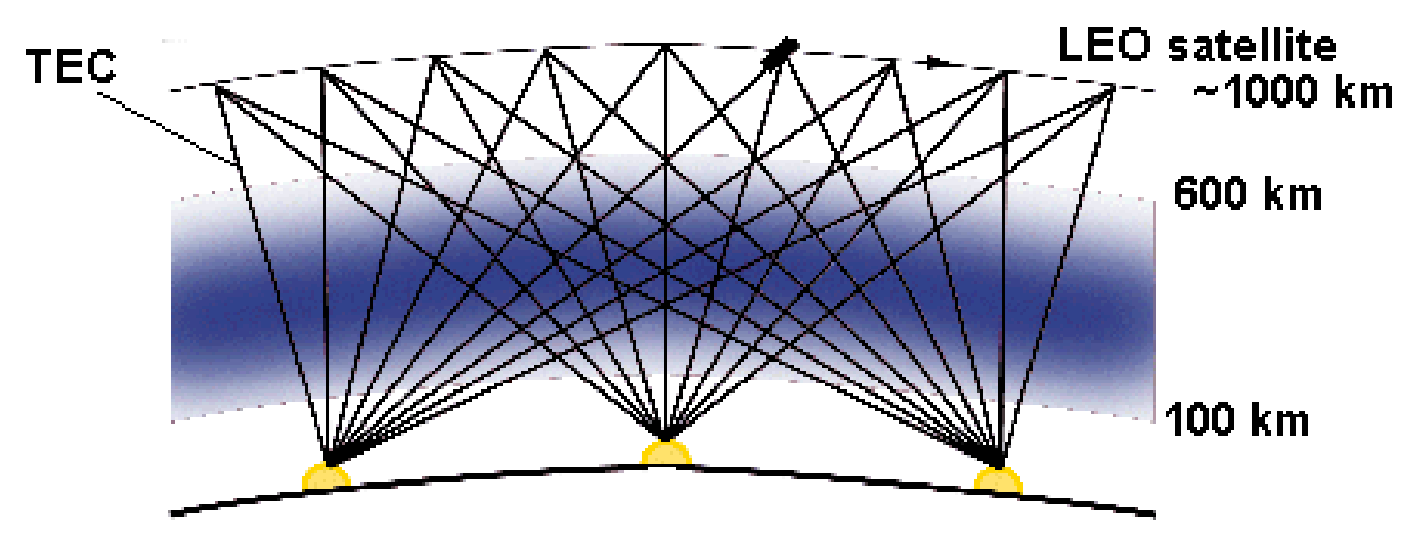

Receiver

Fig. 1. The geometry for ionospheric tomographic imaging, showing the ray paths between a low orbiting satellite and ground receiving stations.

which simultaneously observe the LEO satellite, at an altitude of $\sim 1000 \mathrm{~km}$ from the ground. The total duration of the satellite passes is typically $\sim 10-20$ minutes. In the present study, five receiver locations are considered at $8^{\circ} \mathrm{N}, 13^{\circ} \mathrm{N}$, $17^{\circ} \mathrm{N}, 23^{\circ} \mathrm{N}$ and $28^{\circ} \mathrm{N}$ latitudes. These approximately correspond to the latitudes of the Indian tomography network stations. For the forward modelling, the ionospheric density is assumed to be piecewise constant. For our simulations, geocentric circular grids, having a resolution of $2^{\circ}$ (in latitude) $\times 50 \mathrm{~km}$ (in altitude), are used.

Each line connecting the satellite position and the ground receiver represents one TEC measurement along the line of sight. When all three receivers simultaneously observe the satellite, as represented by lines connecting satellite and receivers, the region having the maximum number of ray path intersections signifies maximum TEC information along different directions. The TEC information content decreases as the number of intersecting ray paths decrease in the latitudealtitude space. In the present study, this space spans from $7^{\circ} \mathrm{N}$ to $29^{\circ} \mathrm{N}$ in latitude and 100 to $550 \mathrm{~km}$ in altitude. The modelling grid is superimposed on the measurement space defined by the satellite and receiver configuration. As mentioned earlier, electron densities are assumed to be constant within each grid. The slant TEC is the sum of the small ray elements within a grid multiplied by the electron densities in the corresponding grid, along each line of sight. Using this information, the electron density distribution in the region is reconstructed.

In our forward model estimations, the IRI model is used to generate the background ionospheric densities over which various structures are artificially introduced. These structures include electron density enhancements and depletions with various latitudinal extents. In fact, these structures are often present in the ionosphere over equatorial and low latitudes. Three distinct ionospheric conditions are considered for inversion in the present study. As mentioned earlier, the forward model to compute the data (TEC's) basically uses the piecewise constant discretization approach. The TEC samples were calculated for every 3-sec interval, to obtain a proper sampling, and also to ensure enough intersecting ray paths through grids. This is quite a realistic sampling, because for the Indian tomography experiment all the receivers have a scan rate of 100 samples per second. This means that a proper time averaging can be performed, which can obviously increase the quality of the data. The same resolution of $2^{\circ} \times 50 \mathrm{~km}$ is preserved for reconstruction also. As a first simulation step, the input TECs are assumed to be representing the absolute TECs along the slant path, connecting the satellite position and the receiver without any measurement error. The SVD algorithm is used for all reconstructions, which do not require any start profile. This ensures the elimination of the possibility of errors due to improper initial profiles.

\section{Results and discussion}

\subsection{Case 1: a geomagnetically quiet ionosphere}

The background ionospheric density for each grid point between $7^{\circ} \mathrm{N}$ to $29^{\circ} \mathrm{N}$ latitude and 100 to $550 \mathrm{~km}$ in altitude, for a geomagnetically quiet ionosphere, are derived using the IRI model. The IRI model is run for 12:00 local time, assuming a sunspot number of 100 . Though the IRI for these conditions predicts a minor increase in the electron density around $17-22^{\circ} \mathrm{N}$ latitude, the latitudinal variation of electron density predicted by the IRI model is too small to represent the EIA associated density enhancements. Therefore, this prediction (Fig. 2a) represents a geomagnetically quiet, low-latitude ionosphere without any major structures present therein.

Figure $2 \mathrm{~b}$ shows its reconstruction using the SVD algorithm. It is seen that there is a fairly good match among these two in retaining the latitudinal as well as altitudinal extents of the electron density distribution in the reconstruction. The maximum difference from the input distribution, 
(a)

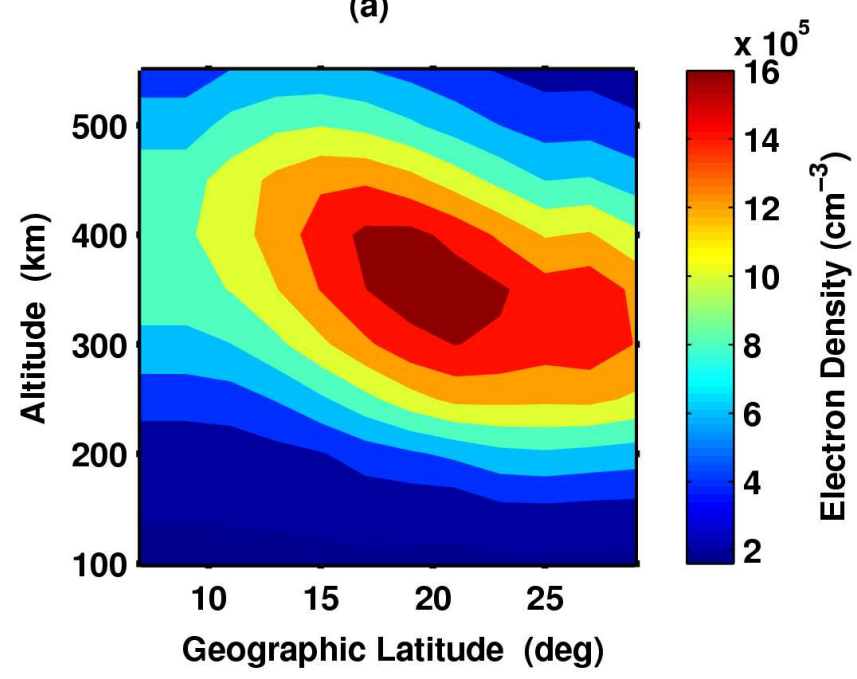

(b)

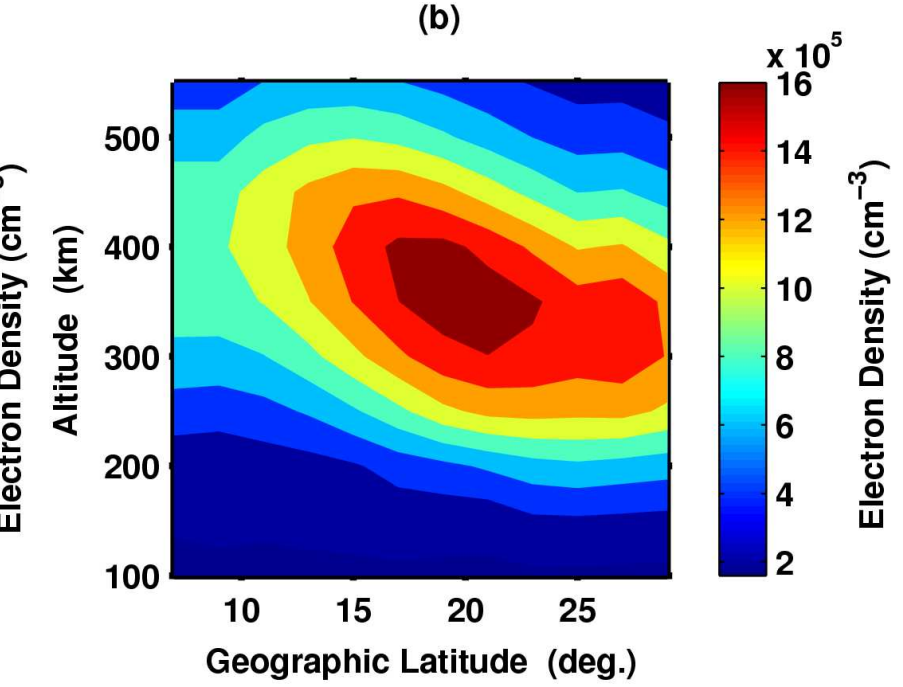

Fig. 2. (a) The model ionosphere used for generating TECs. (b) The tomographic reconstruction corresponding to Fig. 2a.

(a)

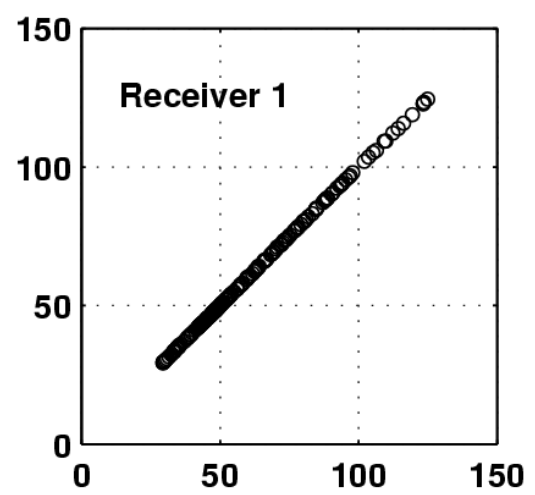

(b)

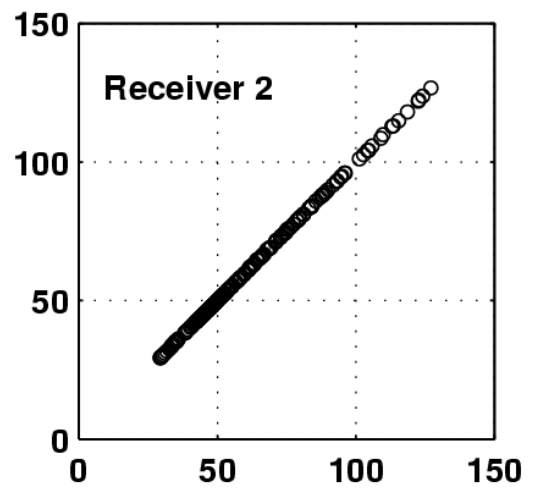

(c)

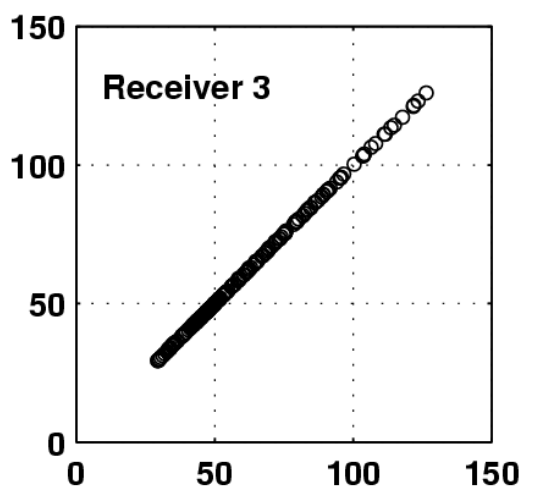

(d)

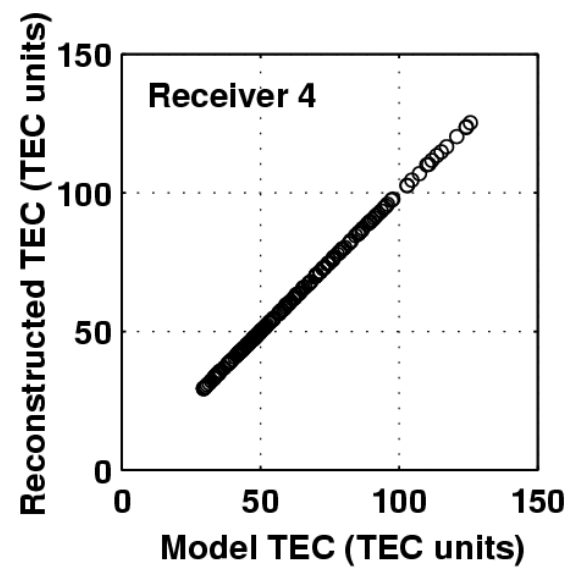

(e)

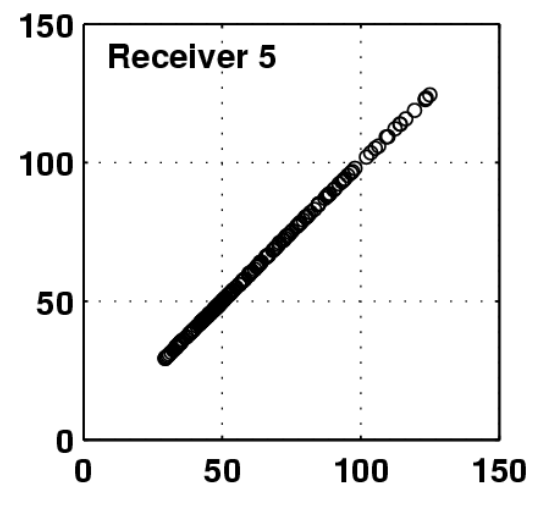

Fig. 3. The input (model) TEC values vs. the reconstructed TEC values for the five receiver locations. 
(a)

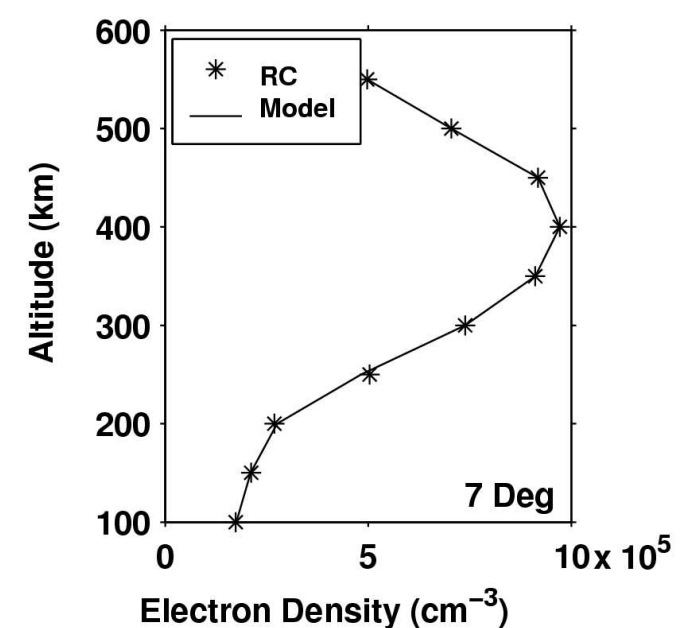

(b)

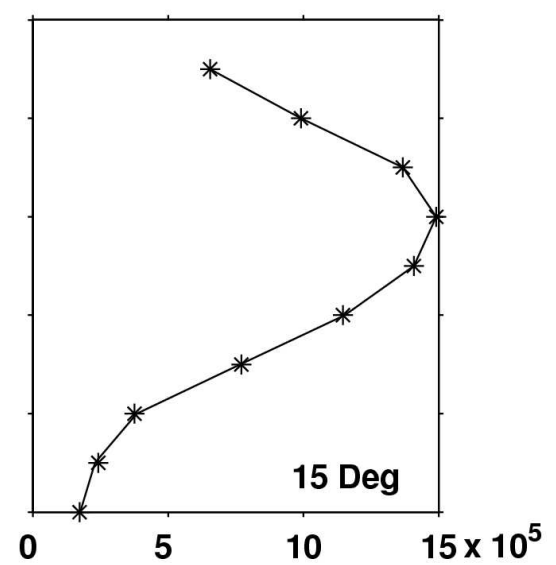

(c)

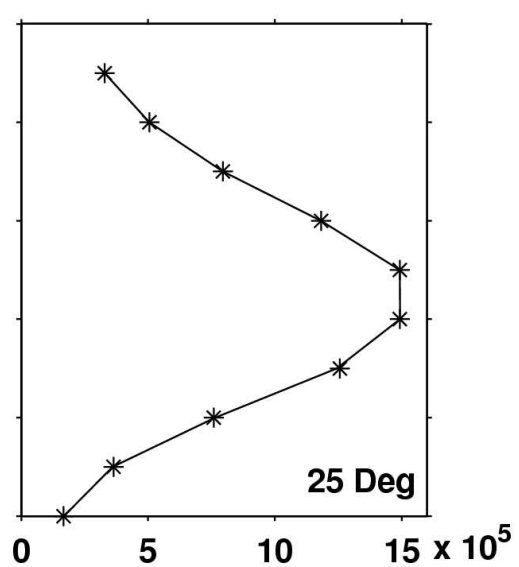

Fig. 4. Three typical vertical profiles. The continuous curve represents the model electron density profiles and the points are the result of the tomographic inversion.
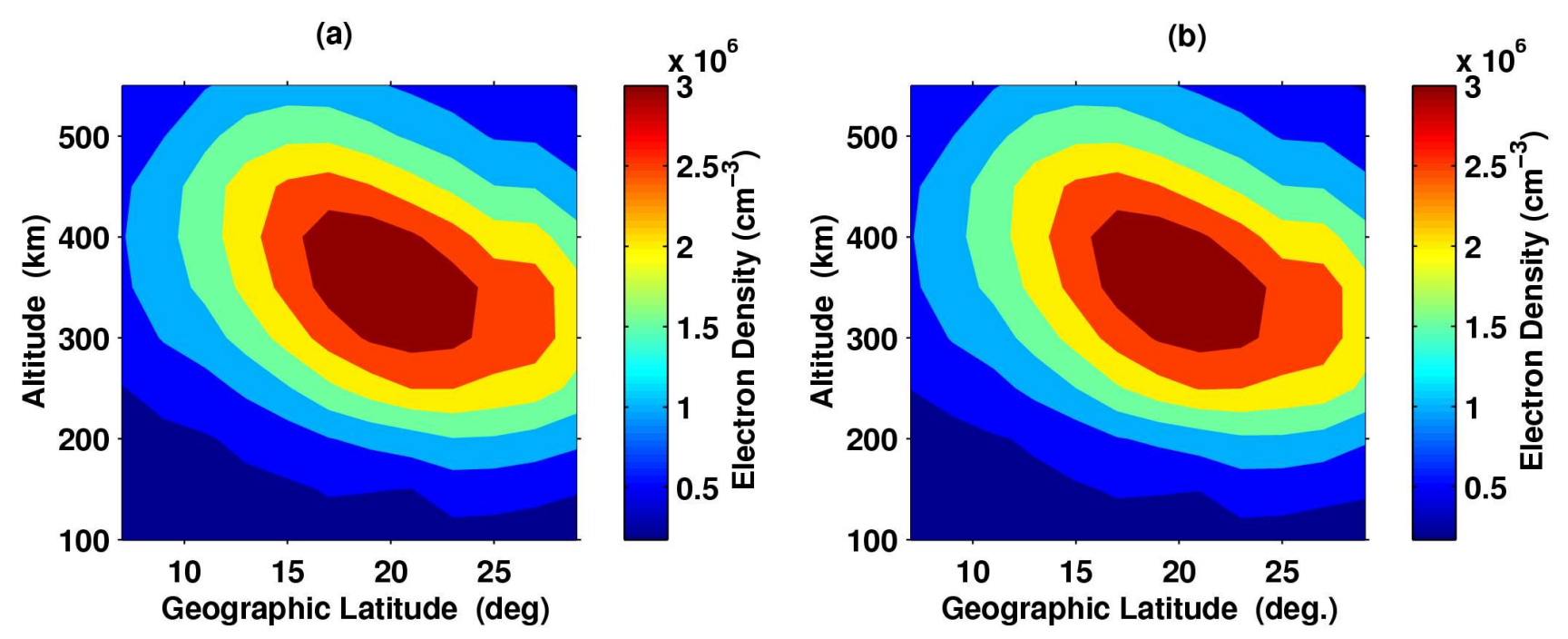

Fig. 5. (a) Same as Fig. 2a but with an enhanced electron density. (b) Same as Fig. 2b but with an enhanced electron density.

i.e. Fig. $2 a$, is less than $1 \%$. Such a small deviation in the reconstruction in this case is due to the fact that the input data is completely error free. Further, the assumption that all the ground-based receivers are receiving satellite signals simultaneously ensures that the geometry has a maximum number of ray path intersections.

However, for checking the accuracy of reconstructions, we use two criteria. First, the reconstructed ionosphere is checked for consistency with the input ionosphere data. For this, TECs are again back calculated from the reconstructed image. Figure $3 \mathrm{a}-\mathrm{e}$ shows the plots of input TECs vs. reconstructed TECs for all five receivers. It is clear that the reconstructed TECs agree well with the input TECs. This proves that the solution obtained using SVD is consistent with the forward model. As a second criteria, the reconstructed ver- tical ionospheric density profiles are compared with the IRI profiles at different latitudes. Figure $4 a-c$ shows three typical input IRI predicted vertical density profiles and their reconstructions. This comparison clearly demonstrates that the two agree well, with deviations being less than $1 \%$ at the altitudes of peak electron density. This could be obtained when the satellite-receiver chain geometry is chosen to have a sufficiently large number of intersecting ray paths.

The effects of errors in the forward model estimated TECs, used for the ionospheric reconstruction on the reconstructed electron density distribution, are dealt with and discussed later. 
(a)

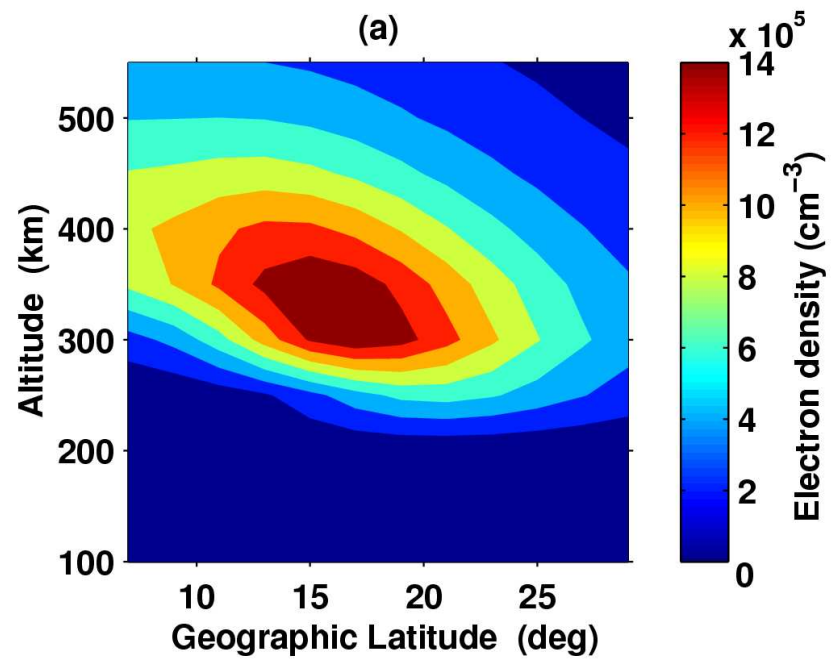

(c)

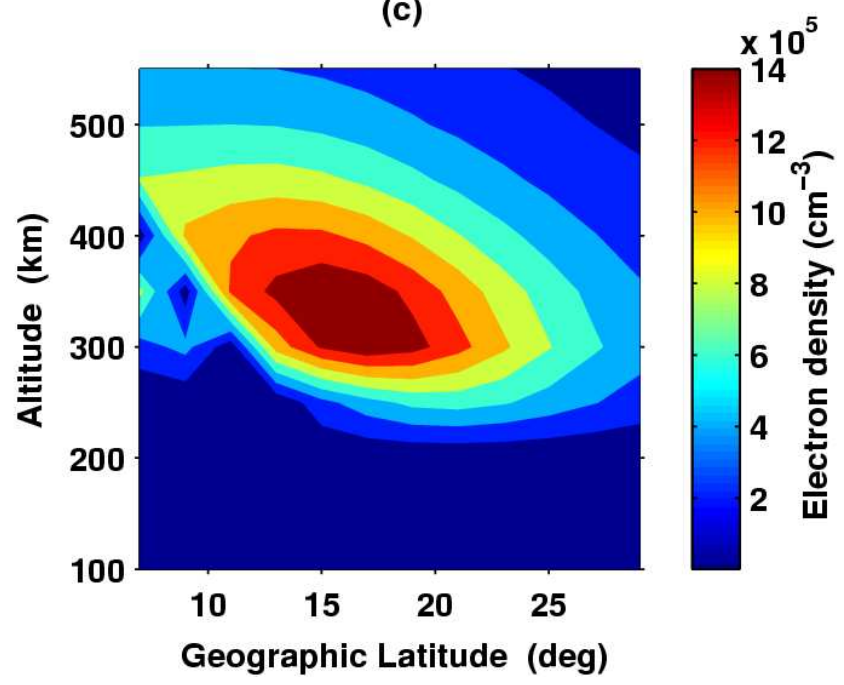

(b)

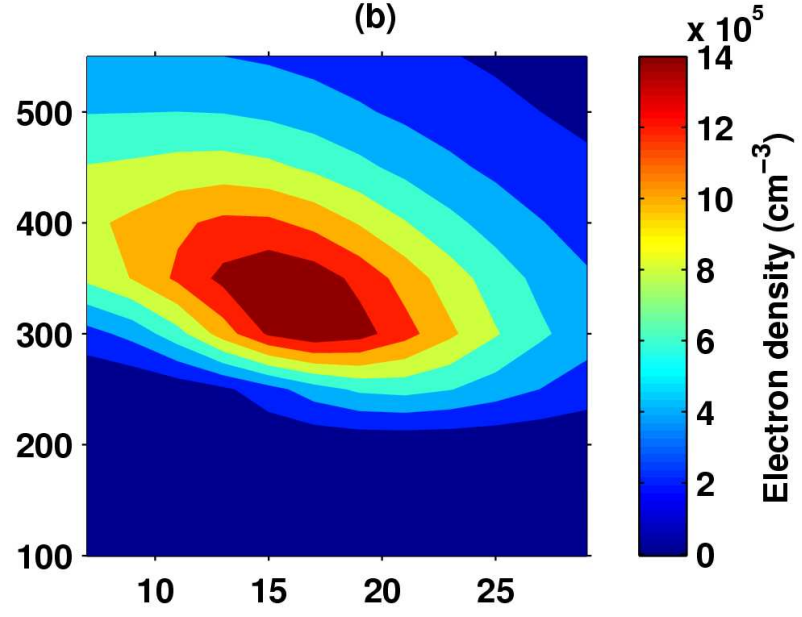

(d)

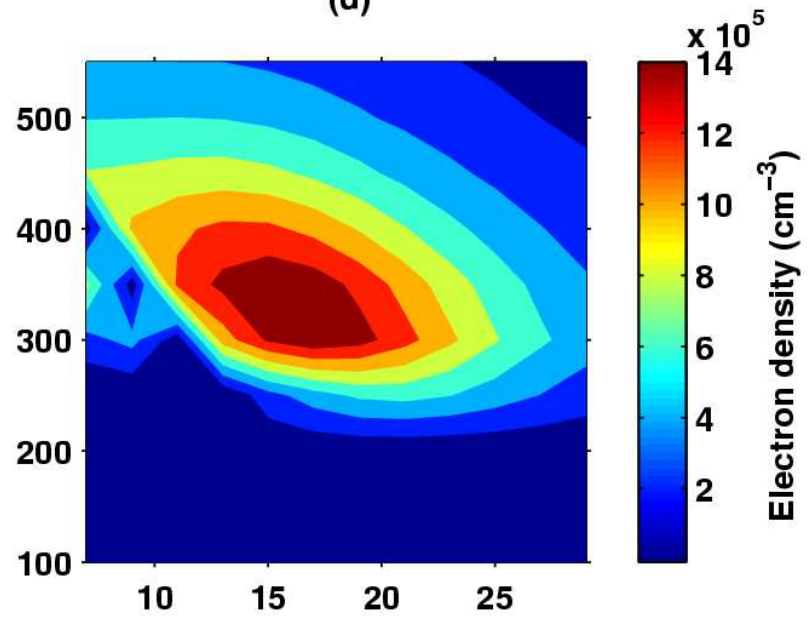

Fig. 6. (a, b) The applied model ionospheres with plasma bubbles at different altitudes. (c, d) The corresponding tomographic inversions.

5.2 Case 2: a geomagnetically quiet ionosphere with Equatorial Ionization Anomaly

As mentioned above, the IRI does not predict the presence of EIA over the equatorial and low-latitude ionosphere realistically. The enhancement seen in the IRI predicted density is not a true indicator of the presence of EIA. Therefore, the latitudinal distribution of ionospheric density typically associated with EIA over low latitudes is simulated and superimposed on the IRI predicted densities for the geomagnetic conditions similar to Case 1. Thus, the obtained ionospheric density distribution that is used in the forward estimations of TECs is exhibited in Figure 5a. This figure exhibits the EIA with enhanced electron density at $\sim 20^{\circ}$, i.e. the crest location, and trough located at $\sim 7^{\circ}$ (geographic latitudes). These variations on electron density in the presence of EIA are quite realistic, for high solar activity periods. EIA variabilities of this kind have been shown to be normally present in the region of our interest, i.e. equatorial and low-latitude region (RaghavaRao et al., 1988, and references therein). In fact, recent LITN observations show that, on a typical day, after the formation of crest at 09:00 LT, it moves poleward in the next two hours with a speed of about $1^{\circ}$ per hour as it intensifies. In the evening hours, it starts to weaken as it recedes equatorward with a speed of $1^{\circ}$ per hour (Andreeva et al., 2000).

Figure $5 \mathrm{~b}$ shows its reconstruction which agrees well with the model input i.e. Figure 5a. The maximum deviation from the model's vertical profile is $\sim 3 \%$. In a study concerning ionospheric tomography, Andreeva et al., (2000) showed that the EIA crest concentrations can vary day-to-day by $60 \%$ in an epoch. In this context, since the vertical profile estimates at the crest location agree within $3 \%$ in the present study, it is possible to investigate the day-to-day variations of crest concentrations with this experimental configuration. Nonetheless, the accuracy in the crest location is determined by the horizontal grid resolution, i.e. $2^{\circ}$ in the present study. 


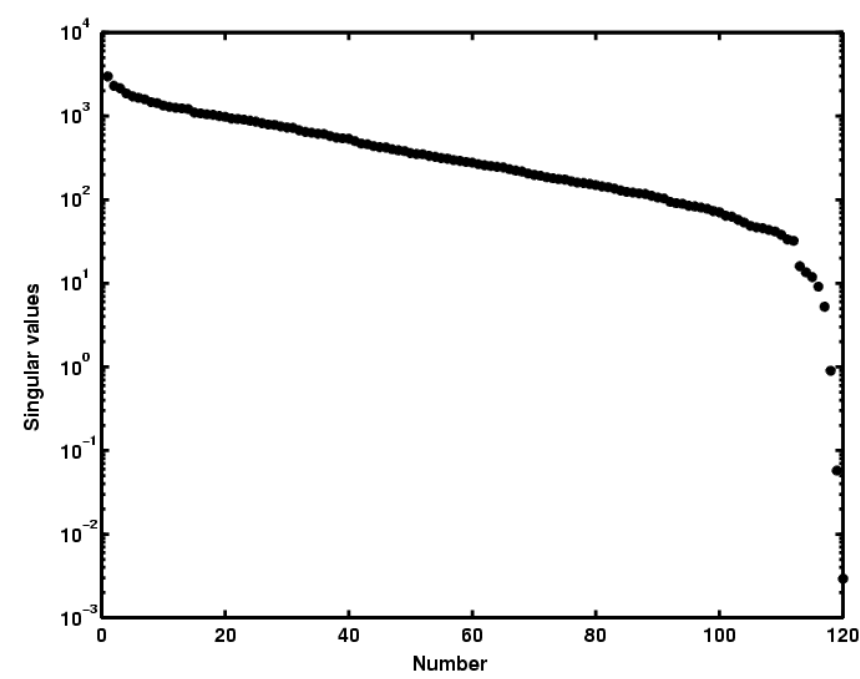

Fig. 7. Distribution of singular values for the geometric matrix A.

Moreover, with the long receiver chain of Indian tomography experiments that covers the typical EIA region, it will be possible to image the movement of EIA during the course of a day.

\subsection{Case 3: plasma bubbles}

Equatorial bubbles (ESF) are the depletions in equatorial $\mathrm{F}$ region plasma that occur after sunset on the bottom side of the F layer and rise to high altitudes. Observing these bubbles is difficult, because they are unpredictable in the time and location of occurrence, and are highly variable in latitudinal and longitudinal extents, as well as in drift velocity and life time (Whalen, 1997). It is seen that the polarization electric field associated with the ESF irregularities can map for long distances along the magnetic field and can thereby influence even the lower altitude plasma (Vickrey et al., 1984).

To understand the ability to reproduce such bubbles by the present tomographic network of receivers, we have simulated plasma bubbles at different altitudes. Figures $6 \mathrm{a}$ and $6 \mathrm{c}$ show the model surfaces with depletions in electron densities. The IRI model is run for 20:00 local time, giving typical sunspot maximum conditions. This represents a typical background electron density distribution of nighttime ionosphere. For Fig. 6a, a small depletion of $30 \%$ is introduced at $250 \mathrm{~km}$, at the $7^{\circ}-9^{\circ}$ grid, and then it is mapped to the off-equatorial latitudes following Farley's equation that describes the mapping of ionospheric structures along the magnetic field lines (Farley, 1960). Similarly, for Fig. 6c, a depletion of $~ 90 \%$ is introduced at $400 \mathrm{~km}$, at the $7^{\circ}-9^{\circ}$ grid, and then it is mapped to the off-equatorial latitudes.

Figures $6 \mathrm{~b}$, and $6 \mathrm{~d}$ show their reconstructions, respectively. It is clear that the present network is able to image the ionosphere with such bubbles present at different altitudes. In the present case, with error free TEC values, the deviations are within $1 \%$. However, the presence of larger errors can make the reconstruction unable to represent the smaller depletions. The effect of errors in such situations is discussed later. Apart from equatorial bubbles, we come across plasma density depletions, during magnetic storms also, which were observed in the experiments of the LITN. It has been observed during a moderate magnetic strom that the steep density gradients in the electron densitites, within a span of $2^{\circ}$, can differ by a factor ranging from few times to about 1 order of magnitude at various regions of the equatorial ionosphere Ji-Sheng et al., 2000). Our study shows that with the present receiver configuration, it is possible to image such gradients also.

\section{Error analysis}

In actual experimental situations, the observed data contain different kinds of errors. For the tomography problem, the main sources of errors in TEC's are (i) the unknown constants in the TEC estimates, (ii) measurement errors and (iii) discretization errors. The unknown constants in TEC arise because of two reasons, (i) the receiver biases and (ii) the incorrect estimation of the cycles in the phase measurement. The unknown constants are those that get added up as a constant to each measurement, either due to receiver errors or due to the unknown initial phases or $2 \mathrm{n} \pi$ ambiguities in the differential Doppler measurements. These can be resolved to an extent by having independent ionosonde measurements at the receiver latitudes.

The ionosonde provides electron density profiles only up to the altitude of maximum electron density (bottom-side profiles). This would give vertical TEC estimates up to the altitude of maximum electron density. The topside profiles are estimated from models and used for obtaining vertical TEC estimates for the topside. A combination of these two gives the vertical TEC measurement at the receiver location. This is used for the estimation of the unknown constant in TEC in the differential Doppler measurement. Typically, the measured slant TECs are of the order of a few tens of TEC units. Considering an average value of 50 TEC units as a typical measurement, the error of $1 \%$ in TEC gets translated to an error of 8 radians. This corresponds to approximately one cycle in the phase measurement. In our simulations, the errors in the TECs range from 1-5\%, corresponding to an error of a few radians in phase estimations, which are quite realistic. For example, the bias errors added are 1\% and 5\% of the minimum TEC (which is $\sim 25$ TECU in the first case), so the errors correspond to 4 radians and 20 radians of phase error.

The random errors generally occur due to the sudden loss of data, by discontinuities in the differential phase measurements that introduce the phase measurement errors for a short duration. These can be clearly seen in the phase data itself. Such data can be rejected, or if the discontinuity is for a short duration the data can be linearly interpolated, without much effect (Leitinger, 1994). Similar to the case of bias errors, the errors added in this case also correspond to errors up to 

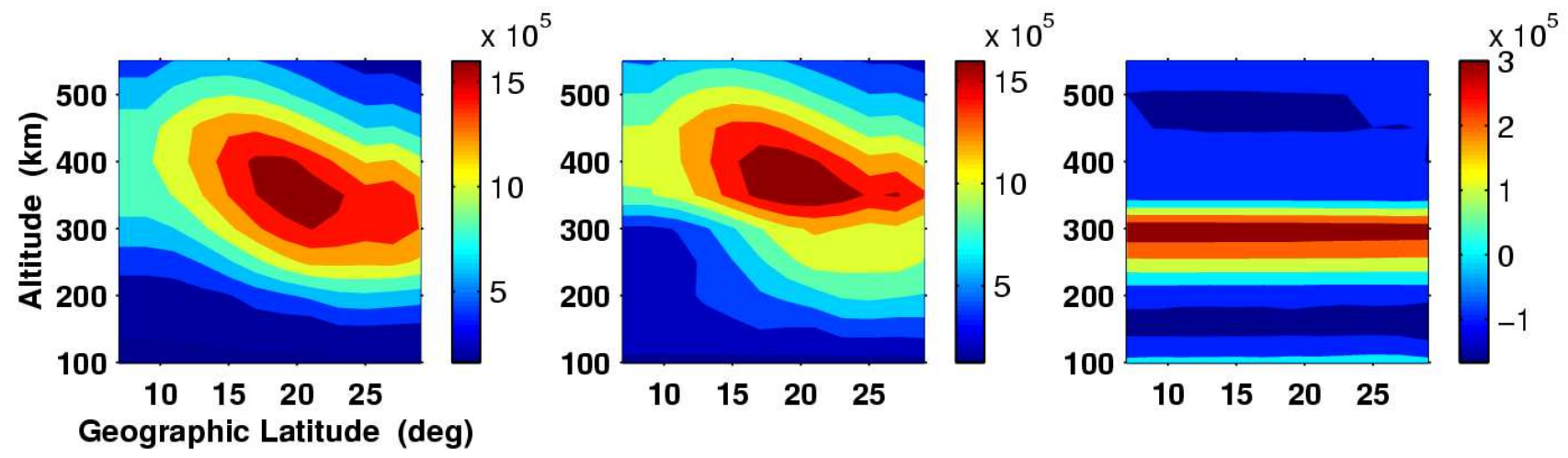

Fig. 8. (a) The model ionosphere for error analysis. (b) The reconstructed image, with a bias error of $1 \%$ of the minimum TEC when the 3 smallest singular values are omitted from the solution. (c) The difference from the model.

a few radians in phase measurement, which is quite possible in real situations.

The next source of error comes from the discretization used for the reconstruction. This is because the actual distribution of electron density in the distribution is not discrete, but continuous. So, the variations in the actual ionosphere are continuous rather than discrete. All reconstruction algorithms will be unable to reconstruct such continuous variations, so the image accuracies will be limited by the grid size chosen for reconstruction. To quantify such errors, the TECs should be calculated using integrals, which represent the actual ionosphere. This error is inherently associated with the grid geometry of the reconstruction, which can be reduced by reducing the size of each pixel (Kunitake et al., 1995).

We have estimated the effect of bias errors, the random errors and the discretization errors on the accuracies of the reconstructed images separately. As mentioned earlier, the truncation of the singular values are extremely important, when the data is erroneous. Figure 7 shows the distribution of all the singular values of the geometry matrix used.

\section{Bias errors and random errors}

Firstly, the geomangentically quiet ionosphere without any major structures is considered for error analysis (same as Fig. 2a). Figure 8 a shows this model. The TECs are estimated from this model and various biases are added to those values. For example, Fig. $8 \mathrm{~b}$ shows the reconstructed image when a bias error of $1 \%$ of the minimum TEC is added to the input data. For obtaining this image, the smallest 3 singular values are omitted from the solution. If we include all the singular values, it is seen that the reconstructed image is not able to reproduce the input electron density distribution (not illustrated). Figure $8 \mathrm{c}$ shows the difference between input and reconstruction.

Figure 9a shows the model which is the same as the earlier one, but another reconstruction is performed with a bias error of $5 \%$ of the minimum TEC added to the input data. Figure $9 \mathrm{~b}$ shows the reconstruction with the 3 smallest val- ues truncated. We can see that the solution still deviates from the model. Figure 9d shows the image obtained when the 4 smallest singular values are truncated. This can be considered as the best solution, because when more singular values are truncated the solution shows again more deviations. Figures $9 \mathrm{f}$ and $9 \mathrm{~h}$ represent the cases when the 5 smallest and 8 singular values are truncated. Figures $9 \mathrm{c}, \mathrm{e}, \mathrm{g}$ and i show the corresponding differences from the model for these three cases. In Fig. 9b, where some of the smaller singular values with errors are also present, the reconstruction is unable to represent the layered structure. This is because of the error magnification due to the presence of the smaller singular values, which tend to perturb the solutions. The presence of one more singular value makes a significant difference in the reconstruction, as most of the features are not reproduced, neither spatially, nor in magnitudes. In Fig. 9d, also, it can be seen that the peak position, as well as the magnitude of the peak electron density, has changed, where both the latitudinal and altitudinal extents of the peak electron densities differ substantially (as high as $\sim 50 \%$ ). So, it is quite reasonable to attempt another reconstruction with more singular values truncated. But the truncation of more singular values also causes significant deviation from the model. As more and more singular values are truncated, the solution tends to become smoother (Figs. 9f and 9h). This is because the singular values contain the actual information about the system, and in truncating the singular values we are actually avoiding some part of the information also, so there is always an optimal truncation where we can reduce the error magnification to obtain the best solution, which for this particular geometry is 4 .

On similar lines, the effect of random errors in the reconstruction is also estimated. The model used for generating the TEC values is shown in Fig. 10a, which is again the same as Fig. 2a, representing a geomagnetically quiet daytime ionosphere obtained using IRI-90 model without any major structures present therein. Random numbers ranging from $0.001 \%$ to $1 \%$ of the average TEC value are added to the forward model estimated TECs as errors. This corresponds to the situation where some of the observations are 


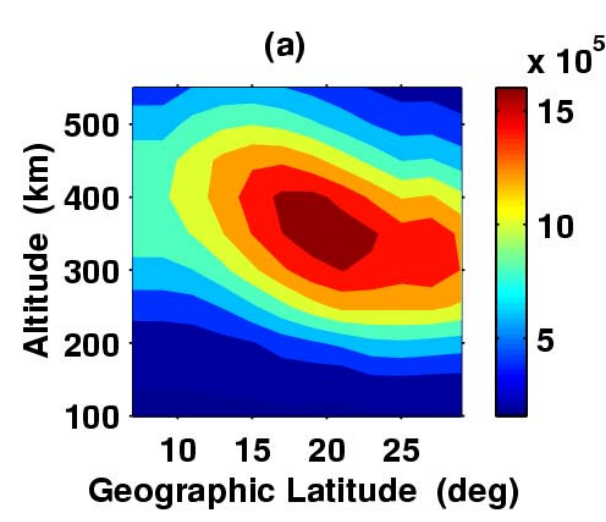

Geographic Latitude (deg)

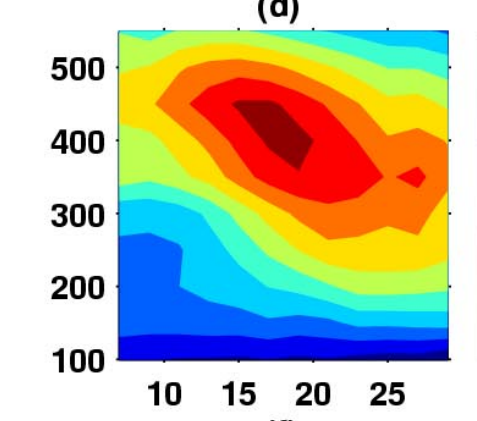

(f)

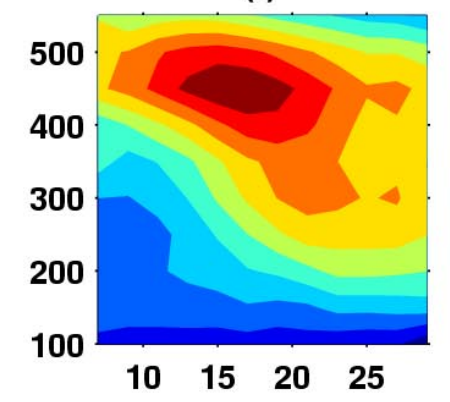

(h)

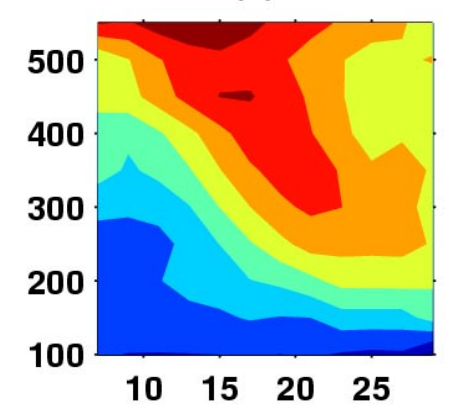

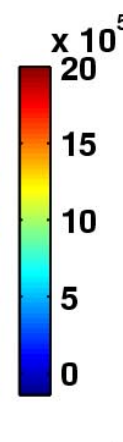

$\times 10^{5}$

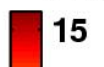

15

$$
3
$$

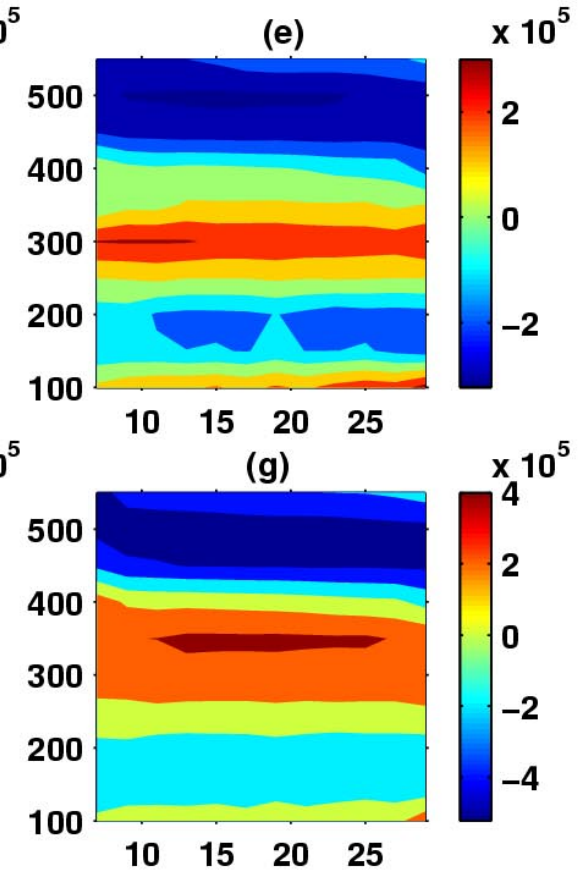

(c)

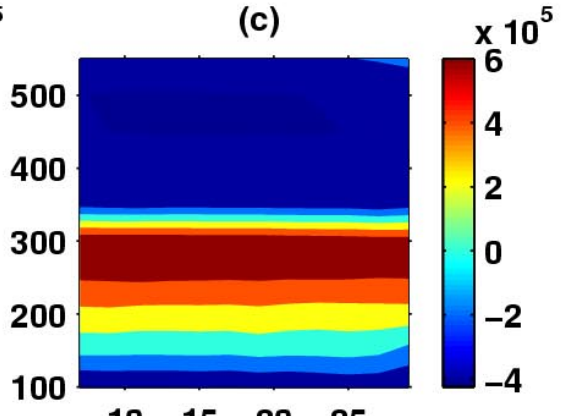

$\begin{array}{lllll}10 & 15 & 20 & 25\end{array}$

(e)
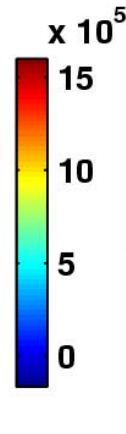

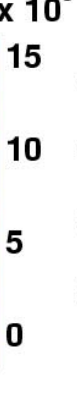



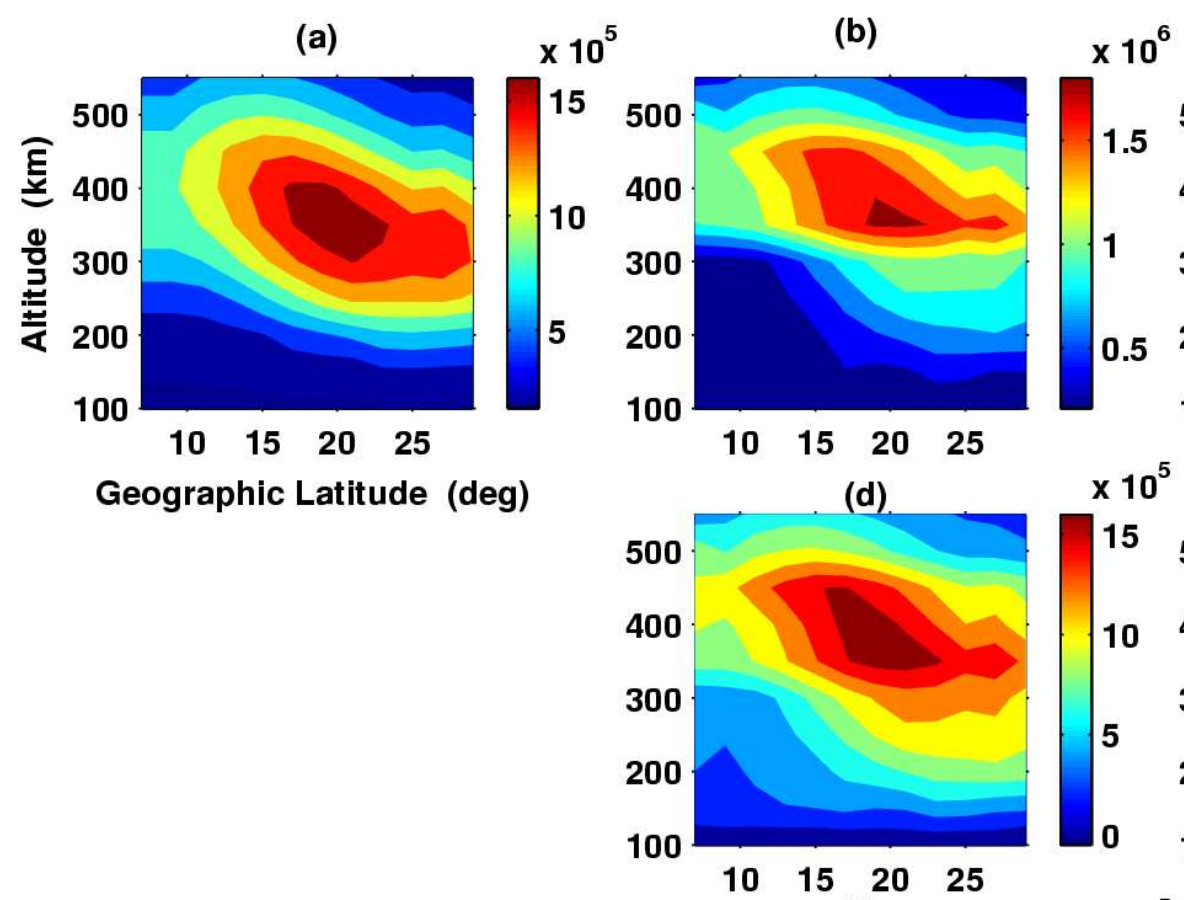

$\mathbf{x} 10^{5}$
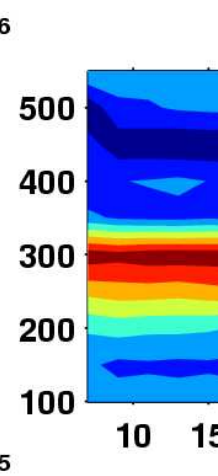

(c)
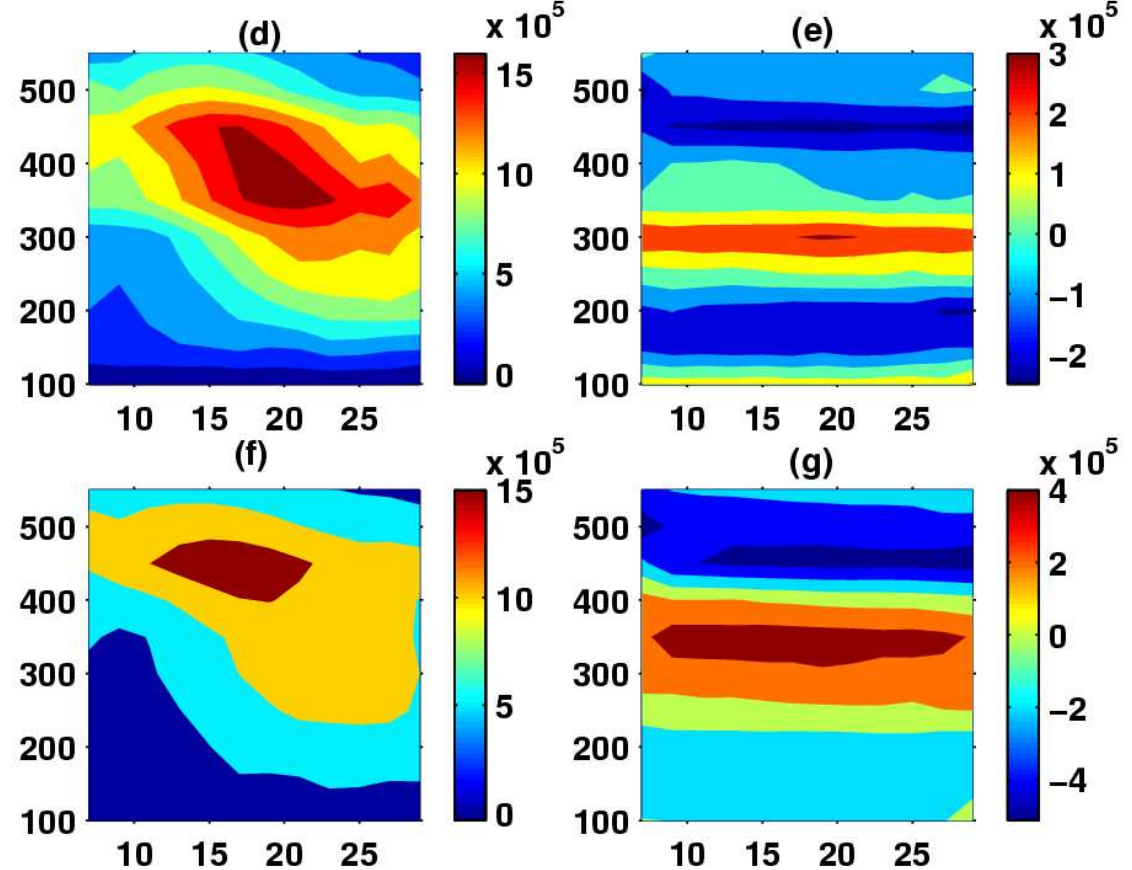

Fig. 10. (a) Same as Fig. 8a. (b) The reconstruction with random errors ranging from $0.001 \%$ to $1 \%$ of the average TEC added to input data and with the 3 lowest singular values truncated. (d) The reconstruction with random errors ranging from $0.001 \%$ to $1 \%$ of the average TEC added to input data and with the 4 lowest singular values truncated. (f) The reconstruction with random errors ranging from $0.001 \%$ to $1 \%$ of the average TEC added to input data and with the 5 lowest singular values truncated. (c, e, g) The corresponding differences from the model in Fig. 10a.

It should be mentioned here that random errors can pose a serious problem in the accuracy of reconstruction, and such errors should be identified and tackled before using the data for reconstruction. This is necessary because in an actual case, random errors can appear with any magnitude, and the presence of such errors, if overlooked, can give wrong solutions. But if the random errors can be identified in the raw data itself, such errors in reconstructions can be avoided.

As the CRABEX aims at imaging the equatorial and lowlatitude ionosphere, which is replete with processes like EIA and plasma bubble, such cases are also used for the present study. Figure 11a represents the model ionosphere, with EIA. Figure $11 \mathrm{~b}$ is its reconstruction with $1 \%$ of the minimum TEC added to the data as bias error. Figure $11 \mathrm{~d}$ is a similar case with $5 \%$ of the minimum TEC as bias error. Figure $11 \mathrm{f}$ is the reconstruction, with random errors of magnitude ranging from $0.001 \%$ to $1 \%$ of the average TEC, added to the data.
Figures 11c, 11e and 11g are the corresponding differences from the model. Here the reconstruction is shown with optimal truncation only, i.e. in all these cases the four smallest singular values are truncated. The effect of truncating more or less the number of singular values is very similar to that of the earlier case, i.e. model ionosphere without any major structures present therein. Another striking aspect is that the image accuracies are much higher than the previous case (maximum deviations are of the order of $15 \%-25 \%$ ). This means that the present network is able to image the EIA and the variability associated with it quite effectively. Or, in general, we can say that large-scale structures can be imaged with reasonable accuracies using tomography techniques.

But when we have to image the ionosphere with structures having relatively smaller density gradients, the errors pose many severe problems. This can be observed in a simulation where we have tried to reconstruct a small plasma bubble 

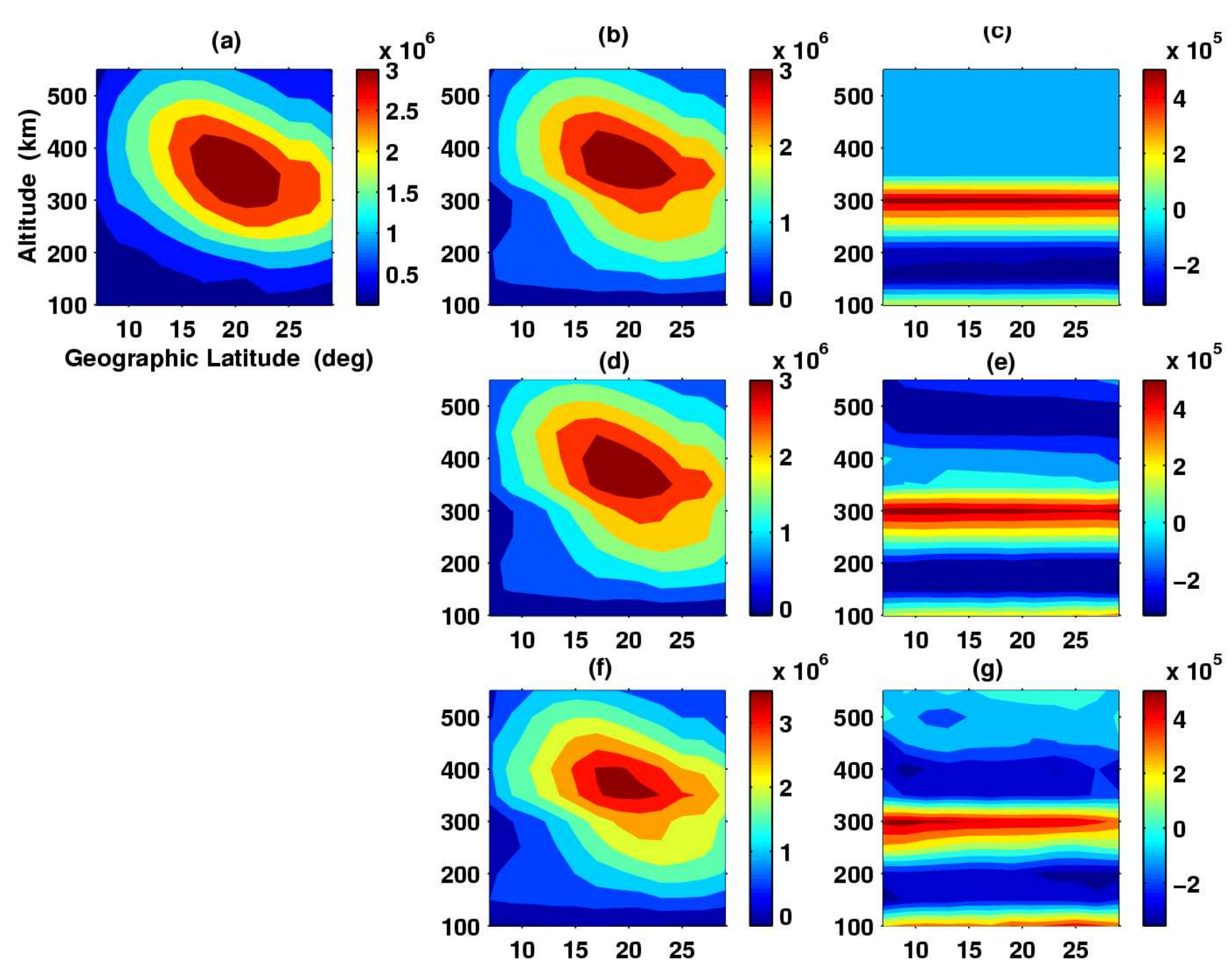

Fig. 11. (a) Model ionosphere with an enhanced electron density. (b) The reconstructed image with a bias error of $1 \%$ of the minimum TEC when the 3 smallest singular values are omitted from the solution. (d) Reconstruction with a bias error of 5\% of the minimum TEC when the 4 smallest singular values are truncated. (f) The reconstruction with random errors ranging from $0.001 \%$ to $1 \%$ of the average TEC added to input data and with the 5 lowest singular values truncated. $(\mathbf{c}, \mathbf{e}, \mathbf{g})$ The corresponding differences from the model in Fig. 11a.

( $\sim 30 \%$ depletion), with errors added to the data. Figure 12a shows the model, and $12 \mathrm{~b}$ is its reconstruction, with $1 \%$ of the minimum TEC added to the data as bias error. Figure $12 \mathrm{~d}$ is another case with $5 \%$ of the minimum TEC as bias error. Figure $12 \mathrm{f}$ is the reconstruction, with random errors added, the maximum being $1 \%$ of the average TEC value. Figures $12 \mathrm{c}, \mathrm{e}, \mathrm{g}$ are the corresponding differences from the model. It can be seen that, even though the depletion in the model is present in the reconstruction, there are additional artifacts in the image. Also, some of the deviations are of the order of the depletion itself, or more, which makes the image reliability substantially low. So, we can say that, if errors are present in the data, the accurate imaging small electron density gradients will be extremely difficult, but large-scale structures and gradients can be quite accurately imaged.

\section{Discretization errors}

So far we have discussed the errors associated with the data. The other important error which limits the accuracy of the tomographic images is the discretization error, which is inherent in the system. As mentioned earlier, the variability in the ionosphere is continuous rather than discrete, and the measured TECs are integrals, so any kind of discretization will have a certain amount of error associated with it. To understand the effect of discretization, we have performed some simulations, first with the ionosphere without much structures, and then with an ionosphere having EIA.

Figure 13a shows the model. For generating the input, each ray path between 100 and $550 \mathrm{~km}$ was divided into 450 (step of $1 \mathrm{~km}$ ) and the TECs are generated as integrals. Reconstruction is performed again, as in the previous cases, for a $50-\mathrm{km}$ vertical grid resolution, and the image obtained is shown in Fig. 13b. We have omitted the three smallest sin- 

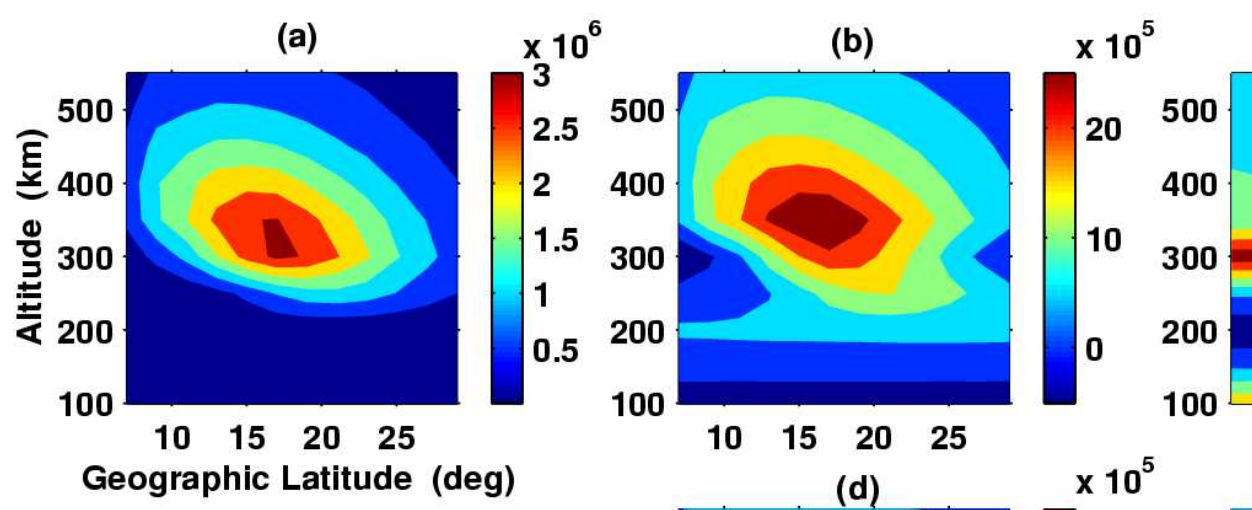

(c)
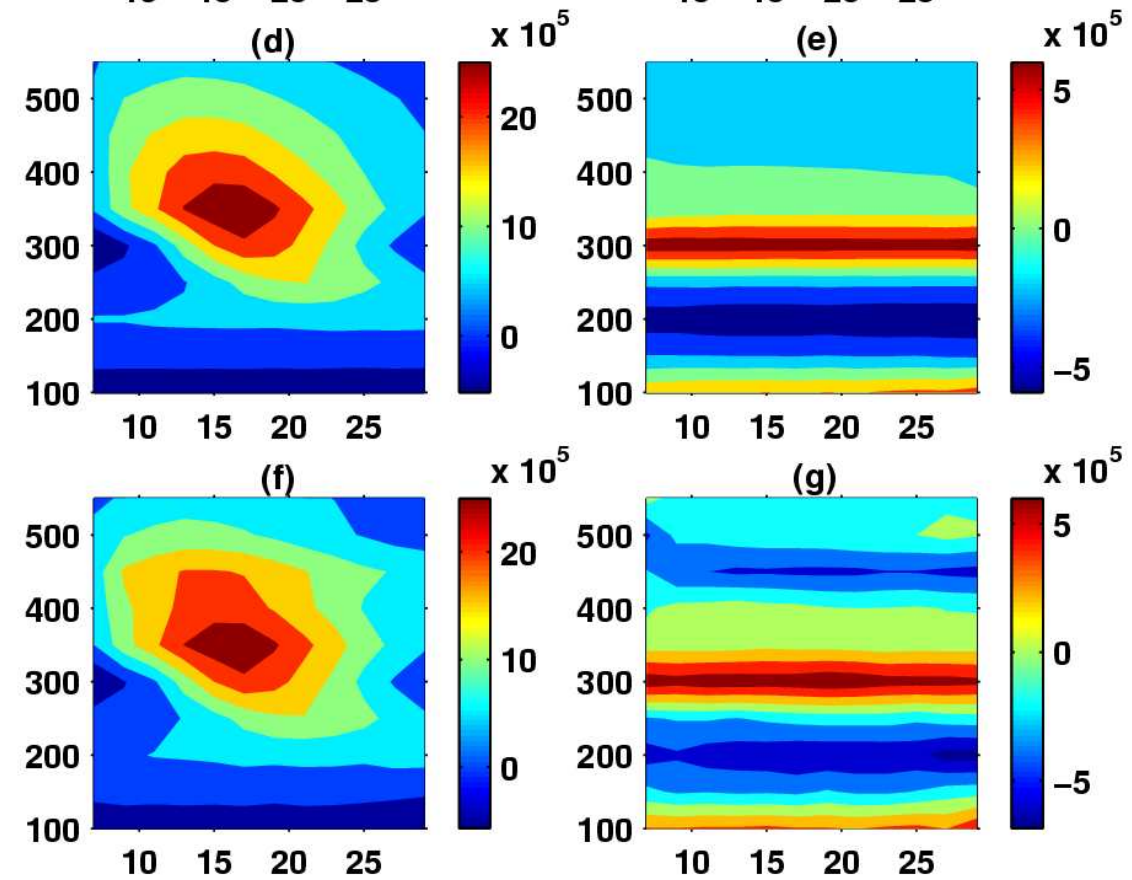

Fig. 12. (a) Model ionosphere with an electron density depletion of $30 \%$. (b) The reconstructed image, with a bias error of $1 \%$ of the minimum TEC when the 3 smallest singular values are omitted from the solution. (d) Reconstruction with a bias error of 5\% of the minimum TEC when the 4 smallest singular values are truncated. (f) The reconstruction with random errors ranging from $0.001 \%$ to $1 \%$ of the average TEC added to input data and with the 4 lowest singular values truncated. (c, e, g) The corresponding differences from the model in Fig. 12a.

gular values in this case, which are seen to be the optimal truncation. If we include more singular values, the solution has larger perturbations, and the solution with a lesser number of singular values tends to be smoother (not illustrated). In a similar manner, we have considered the case of EIA also. Figure 14a shows the model and $14 \mathrm{~b}$ shows the image with optimal truncation, in which we have omitted the three smallest values. It can be seen that for both these cases, the error due to discretization is significant. As discussed in Kunitake et al. (1995), we can attribute this as being due to the approximation in TEC to a finite dimensional expression $Y=A x$. To reduce the discretization error, the size of each pixel should be reduced. This will make the number of pixels much larger; hence, the matrix size will be much larger.

All these errors, when present, generally make the reconstruction inaccurate. However, in actual cases, more than one inversion procedure can be followed and the image ac- curacies can be enhanced by averaging all of the images in the assembly of solutions (Andreeva et al., 2001).

\section{Conclusion}

The present simulation study shows that the SVD technique can be used with certain limitations for tomographic studies. This technique is useful to understand the effect of receiver configuration on the reconstructed ionospheric images. In SVD, the singular values contain the information about the system, and when errors are associated with the data, the presence of smaller singular values amplify the numerical error during inversion, thus causing perturbations in the solution. So, we have to truncate the singular values to obtain the best solution. Including more singular values will cause larger perturbations, while avoiding more singular values tends to yield an over-smooth solution. So there is an 

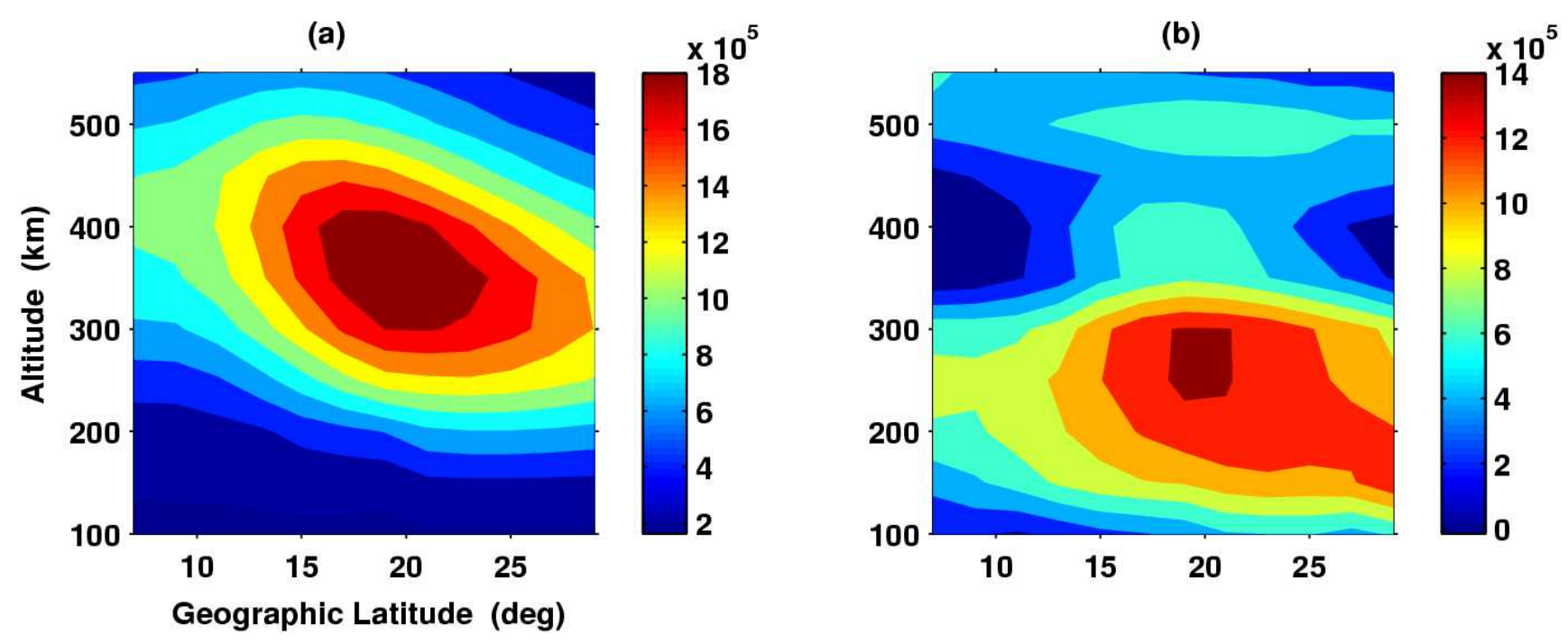

Fig. 13. (a) The model used to study the discretization error. (b) The tomographic reconstruction corresponding to Fig. 13a.
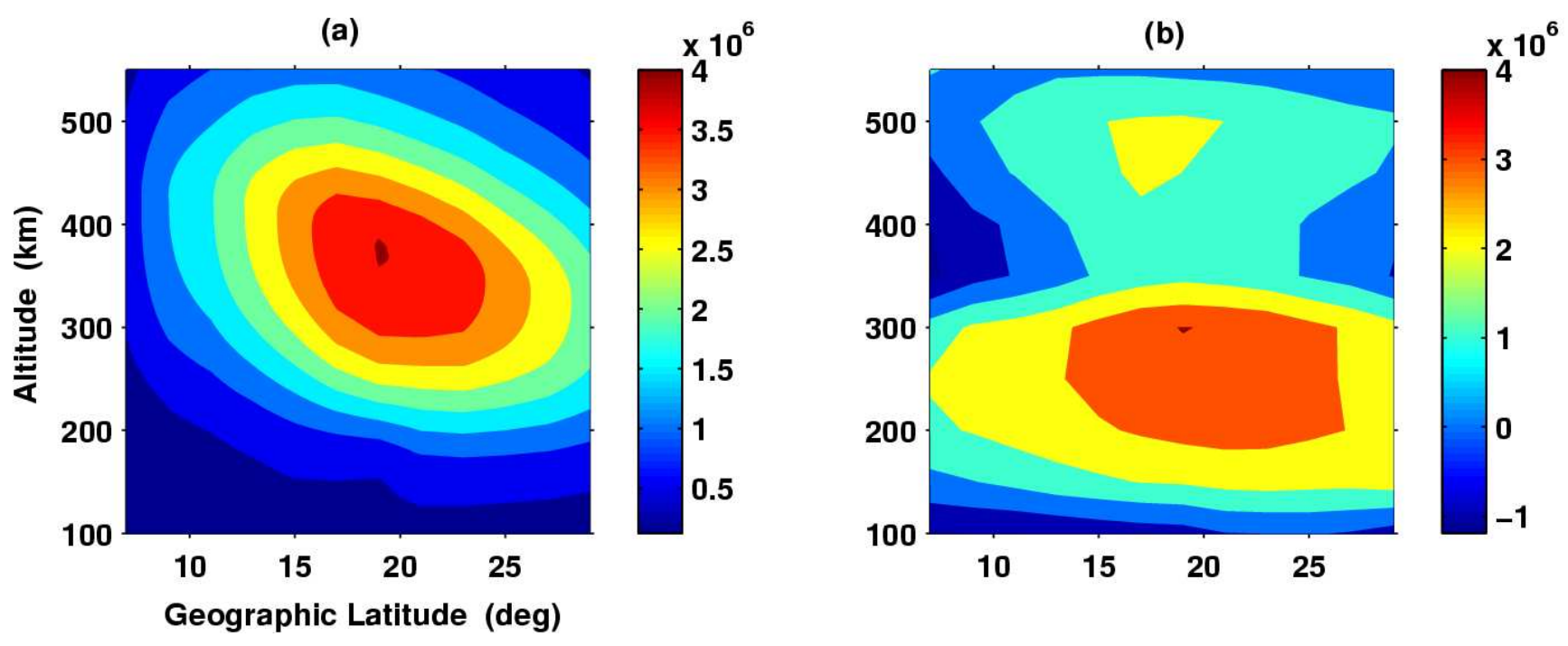

Fig. 14. (a) The model with an enhancement in electron density, used to study the discretization error. (b) The tomographic reconstruction corresponding to Fig. 14a.

optimum value for truncation, which, in the present case, is seen to be the truncation of the 4 smallest singular values for the case of data with errors. The effect of the discretization error is also studied, for which the truncation of the 3 singular values gave the best solution. These simulations are extremely important in view of the experiment, because the data will always contain errors.

As mentioned earlier, in the Indian tomographic network of receivers, the measured data is the differential Doppler, which is proportional to the slant relative TEC. Since we are measuring the relative phase only, the measured phase has an arbitrary starting point. So, we have to add an initial phase to obtain the true relative phase. In practice, this is achieved through independent measurements. However, any inaccurate estimation of the initial phase is a significant source of error. These are the sources of unknown constants or bias errors in the data. The errors due to an unknown initial phase can be minimized effectively using the phase difference methods (Andreeva et al., 1992). In addition to this, there are inherent inaccuracies in measurements because of phase errors in the phase lock loops of the receiver, and the overall phase change due to antenna pattern and noise. These are probably of a magnitude of $2 \pi / 10$ at the $50 \mathrm{MHz}$ level (Leitinger, 1994). This also adds to the errors in the data, most of which are really random in nature. Averaging methods can minimize the random errors in the solution. Even with all these error minimization procedures, the data will still not be completely error free. So, we have estimated the effect of the errors in the accuracies of reconstruction. 
The difference in the reconstruction caused by various errors varies from a few percent to as large as $50 \%$. The bias errors and random errors cause deviations, both in the case of the ionosphere without any major structures, as well as for the case of EIA. The image accuracies are much higher for the case with EIA (maximum deviations are of the order of $15 \%-25 \%$ ). Apart from data errors, the discretization used for the imaging is an inherent source of error. The effect of discretization is also understood using simulations. However, the effect of this error can be minimized by reducing the pixel sizes (Kunitake et al., 1995).

The simulations helped to understand that by using the coherent radio beacon (CRABEX) network of the Indian tomography experiment, it would be possible to obtain accurate images of the large-scale structures like EIA, which are present in the equatorial and low-latitude ionosphere. This unique chain will be able to provide valuable information regarding the crest-to-trough ratio during varying geophysical conditions and the day-to-day variabilities and movement of the anomaly crest. The present network would also be able to image structures like equatorial bubbles, which are often present in the region, provided the processed data (after all error minimization procedures, like unknown constant estimations and averaging) are error free to less than $5 \%$ of the average TEC measurement.

The deviation of the reconstructed image from the model surface is only within $1 \%$ when the data do not contain any kind of errors, and the geometry is the optimum one with maximum number of path- pixel intersections. This means that solutions obtained using SVD are consistent with the data.

The present analysis highlights that tomographic imaging has the potential for evolving as a powerful tool for the investigation of equatorial ionospheric structures and associated dynamics.

Acknowledgements. One of the authors, Smitha V Thampi, gratefully acknowledges the financial support provided by the Indian Space Research Organization through the research fellowship.

Topical Editor M. Lester thanks R. Leitinger and M. Kunitake for their help in evaluating this paper.

\section{References}

Andreeva, E. S., Franke, S. J., Yeh, K. C., Kunitsyn, V. E., and Nesterov, I. A.: On generation of an assembly of images in ionospheric tomography, Radio Sci., 36, 299-309, 2001.

Andreeva, E. S., Franke, S. J., Yeh, K. C., and Kunitsyn, V. E.: Some features of the equatorial anomaly revealed by ionospheric tomography, Geophy. Res. Lett., 27, 2465-2468, 2000.

Andreeva, E. S., Kunitsyn, V. E., and Tereshchenko, E. D.: Phase difference tomography of the ionosphere. Ann. Geophysicae, 10, 849-855, 1992.

Andreeva, E. S., Galinov, A. V., Kunitsyn, V. E., Mel'nichenko, Y. A., Tereshchenkov,E. D., Filimonov, M. A., and Chernyakov, S. M.: Radiotomographic reconstruction of ionization dip in the plasma near the Earth, JETP Lett., 52, 145-148, 1990.
Austen, J. R, Franke, S. J., and Liu, C. H.: Ionospheric imaging using computerized Tomography, Radio Sci., 23, 299-307, 1988.

Bracewell R. N.: Strip integration in radio astronomy, Aus. J. Phys., 9, 198-217, 1956.

Censor, Y.: Finite Series-Expansion Reconstruction methods. Proc. IEEE., 71, 409-419, 1983.

Farley, D. T., Jr.: A theory of electrostatic fields in the ionosphere at nonpolar geomagnetic latitudes, J. Geophys. Res., 65, 869, 1960.

Foster, J. C., Klobuchar, J. A., Kunitsyn, V. E., Tereshchenkov, E. D., Andreeva,E. S., Bounsanto, M. J., Fougere, P., Holt, J. M., Khudukon, B. Z., Pakula, W., and Raymund, T. D.: Russian American tomography experiment, Int. J. Imaging Syst. Techn., 5, 148, 159, 1994.

Fougere, P. F.: Ionospheric radio tomography using maximum entropy, 1. Theory and simulation studies, Radio Sci., 30, 429-444, 1995.

Fremouw, E. J, Secan, J., and Howe, B. M.: Application of stochastic inverse theory to ionospheric tomography, Radio Sci., 27, 721, 1992.

Fremouw, E. J, Secan, J., Bussey, R. M., and Howe, B. M.: A status report on applying discrete inverse theory to ionospheric tomography, Int. J. Imaging Syst. Techno., 5, 97, 1994.

Hajj, G. A., Ibanez-Meier R., Kursinski, E. R., and Romans, L. J.: Imaging the ionosphere with the Global Positioning system,Int. J. Imaging Syst. Techno., 5, 174, 1994.

Ji- Sheng, Xu., Shu- Ying, M. A., Xiong-Bin, W. U, Lin, K. H., and Yeh ,K. C.: Tomographic imaging of low latitude ionosphere response to a magnetic storm, Chinese J. Geophys., 43, 159-166, 2000.

Kersley, L., Pryse, S. E., Walker, I. K., Heaton, J. A. T., Mitchell, C. N., Williams, M. J., and Wilson, C. A.: Imaging of electron density troughs by tomographic techniques, Radio Sci., 32, 16071621, 1997.

Kunitake, M., Ohtaka, K., Maruyama, T., Tokumaru, M., Morioka, A., and Watanbe, S.: Tomographic imaging of the ionosphere over Japan by the modified truncated SVD method, Ann. Geophysicae, 13, 1303-1310, 1995.

Kunitsyn, V. E. and Tereshchenko, E. D.: Radio tomography of the ionosphere, Antenn. Prop. Mag., 34, 22-32, 1994.

Leitinger, R.: Data from orbiting navigation satellites for tomographic reconstruction, J. Imag. Syst. And Technol., 5, 86-96, 1994.

Na. H, Hall, B., and Sutton, E.: Ground station spacing effects in ionospheric tomography. Ann. Geophysicae, 13, 1288-1296, 1995.

Na, H. and Sutton, E.: Resolution analysis of ionospheric tomography systems, Int. J. Imaging Syst. Techno., 5, 169, 1994.

Pakula, W. A., Fougere, P. F., Klobuchar, J. A., Kuenzler, H. J., Buonsanto, M. J., Roth, J. M., Foster, J. C., and Sheehan, R. E.: Tomographic reconstruction of the ionosphere over North America with comparisons to ground based radar, Radio Sci., 30, 89103, 1995.

Press, W. H., Flannery, B. P., Teukolsky, S. A., and Vetterling, W. T., Numerical Recipes (FORTRAN), Cambridge Univ. Press, New York, 1989.

Pryse, S. E., and L. Kersley, A preliminary experimental technique for ionospheric tomography, J. Atmos. Terr. Phys. 54, 10071012, 1992.

Raghava Rao, R., Sridharan, R., Sastri, J. H., Agashe, V. V., Rao, B. C. N., Rao, P. B., and Somayajulu, V. V.: The equatorial ionosphere, in World ionosphere- theremosphere study, WITS Handbook, edited by C. H. Liu and B. Edwards, 1988. 
Raymund, T. D., Pryse, S. E., Heaton, J. A. T., and Kersley, L.: Tomographic reconstruction of ionospheric electron density with European incoherent scatter radar verification, Radio Sci., 28, 811-817, 1993.

Raymund T. D, Austen, J. R., Franke, S. J., Liu, C. H., Klobuchar, J. A., and Stalker, J.: Application of computerized tomography to the investigation of ionospheric structures, Radio Sci., 25, 771789, 1990.

Vickrey, J. F., Kelley, M. C., Pfaff, R., and Goldman, S. R.: Lowlatitude image striations associated with bottomside equatorial spread F: observations and theory, J. Geophys. Res., 89, 2955, 1984.

Whalen, J. A.: The equatorial anomaly: Its quantitative relation to equatorial bubbles, bottomside spread $\mathrm{F}$ and ExB drift velocity during a month at solar maximum, J. Geophys. Res., 106, 29 125-29 132, 2001.
Whalen, J. A.: Equatorial bubbles observed at the north and south anomaly crests: Dependence on season, local time and dip latitude, Radio Sci., 32, 4, 1559-1566, 1997.

Yeh, H. C., Franke, S. J., Yeh, K. C., Liu, C. H., Raymund, T. D., Chen, H. H., Izotov, A. V., Liu, J. Y., Wu, J., Lin, K. H. and Chen, S. W.: Low- latitude ionospheric tomography network along Taiwan meridian, in Low latitude ionospheric physics, edited by FuShong Kuo, Elsevier Sci, NY, 1994.

Yeh, K. C., Franke, S. J., Andreeva, E. S., and Kunitsyn, V. E.: An investigation of motion of the equatorial anomaly crest, Geophy. Res. Lett., 28, 4517-4520, 2001.

Zhou, C., Fremouw, E. J., and Sahr, J. D: Optimal truncation criterion for application of singular value decomposition to ionospheric tomography, Radio Sci., 34, 155-166, 1999. 